J. Indones. Math. Soc.

Vol. 18, No. 1 (2012), pp. 1-19.

\title{
VERTEX EXPONENTS OF A CLASS OF TWO-COLORED HAMILTONIAN DIGRAPHS
}

\author{
Aghni Syahmarani ${ }^{1}$ and Saib Suwilo ${ }^{2}$ \\ ${ }^{1}$ Department of Mathematics, University of Sumatera Utara, Indonesia \\ Department of Mathematics, University of Sumatera Utara, Indonesia, \\ saib@usu.ac.id
}

\begin{abstract}
A two-colored digraph $D^{(2)}$ is primitive provided there are nonnegative integers $h$ and $k$ such that for each pair of not necessarily distinct vertices $u$ and $v$ in $D^{(2)}$ there exists a $(h, k)$-walk in $D^{(2)}$ from $u$ to $v$. The exponent of a primitive twocolored digraph $D^{(2)}, \exp \left(D^{(2)}\right)$, is the smallest positive integer $h+k$ taken over all such nonnegative integers $h$ and $k$. The exponent of a vertex $v$ in $D^{(2)}$ is the smallest positive integer $s+t$ such that for each vertex $u$ in $D^{(2)}$ there is an $(s, t)$-walk from $v$ to $u$. We study the vertex exponents of primitive two-colored digraphs $L_{n}^{(2)}$ on $n \geq 5$ vertices whose underlying digraph is the Hamiltonian digraph consisting of the cycle $v_{1} \rightarrow v_{n} \rightarrow v_{n-1} \rightarrow \cdots \rightarrow v_{2} \rightarrow v_{1}$ and the arc $v_{1} \rightarrow v_{n-2}$. For such two-colored digraph it is known that $2 n^{2}-6 n+2 \leq \exp \left(L_{n}^{(2)}\right) \leq\left(n^{3}-2 n^{2}+1\right) / 2$. We show that if $\exp \left(L_{n}^{(2)}\right)=\left(n^{3}-2 n^{2}+1\right) / 2$, then its vertex exponents lie on $\left[\left(n^{3}-2 n^{2}-3 n+4\right) / 4,\left(n^{3}-2 n^{2}+3 n+6\right) / 4\right]$ and if $\exp \left(L_{n}^{(2)}\right)=2 n^{2}-6 n+2$, then its vertex exponents lie on $\left[n^{2}-4 n+5, n^{2}-2 n-1\right]$.
\end{abstract}

Key words: Two-colored digraph, primitive digraph, exponent, vertex exponent, hamiltonian digraph.

2000 Mathematics Subject Classification: 05C15, 05C20. 


\begin{abstract}
Abstrak. Digraf dwiwarna $D^{(2)}$ adalah primitif dengan syarat terdapat bilangan bulat nonnegatif $h$ dan $k$ sehingga untuk setiap pasangan yang tidak perlu berbeda titik $u$ dan $v$ di $D^{(2)}$ terdapat sebuah jalan $(h, k)$ di $D^{(2)}$ dari $u$ ke $v$. Eksponen dari primitif digraf dwiwarna $D^{(2)}$, yang dinotasikan dengan $\exp \left(D^{(2)}\right)$, adalah bilangan bulat positif terkecil $h+k$ dari semua jumlahan yang mungkin bilangan bulat nonnegatif $h$ dan $k$. Eksponen dari sebuah titik $v$ di $D^{(2)}$ adalah bilangan bulat positif terkecil $s+t$ sehingga untuk tiap titik $u$ di $D^{(2)}$ terdapat sebuah jalan $(s, t)$ dari $v$ ke $u$. Pada paper ini akan dipelajari eksponen titik dari digraf dwiwarna primitif $L_{n}^{(2)}$ pada $n \geq 5$ titik dengan digraf dasar adalah digraf Hamilton yang memuat lingkaran $v_{1} \rightarrow v_{n} \rightarrow v_{n-1} \rightarrow \cdots \rightarrow v_{2} \rightarrow v_{1}$ dan busur $v_{1} \rightarrow v_{n-2}$. Untuk digraf dwiwarna yang demikian telah diketahui bahwa $2 n^{2}-6 n+2 \leq \exp \left(L_{n}^{(2)}\right) \leq$ $\left(n^{3}-2 n^{2}+1\right) / 2$. Paper ini menunjukkan bahwa jika $\exp \left(L_{n}^{(2)}\right)=\left(n^{3}-2 n^{2}+1\right) / 2$, maka eksponen titiknya berada pada $\left[\left(n^{3}-2 n^{2}-3 n+4\right) / 4,\left(n^{3}-2 n^{2}+3 n+6\right) / 4\right]$ dan jika $\exp \left(L_{n}^{(2)}\right)=2 n^{2}-6 n+2$, maka eksponen titiknya berada pada $\left[n^{2}-4 n+\right.$ $\left.5, n^{2}-2 n-1\right]$.

Kata kunci: Digraf dwiwarna, digraf primitif, eksponen, eksponen titik, digraf Hamilton.
\end{abstract}

\title{
1. Introduction
}

Given a vector $\mathbf{x}$ we use the notation $\mathbf{x} \geq 0$ to show that $\mathbf{x}$ is a nonnegative vector, that is, a vector each of whose entry is nonnegative. Thus for two vectors $\mathbf{x}$ and $\mathbf{y}$, the notion $\mathbf{x} \geq \mathbf{y}$ means that $\mathbf{x}-\mathbf{y} \geq 0$.

A digraph $D$ is strongly connected provided for each pair of vertices $u$ and $v$ in $D$ there is a $u v$-walk from $u$ to $v$. A ministrong digraph is a strongly digraph such that removal of any single arc will result in a not strongly connected digraph. A strongly connected digraph $D$ is primitive provided there exists a positive integer $\ell$ such that for every pair of not necessarily distinct vertices $u$ and $v$ in $D$ there is a walk from $u$ to $v$ of length $\ell$. The smallest of such positive integer $\ell$ is the exponent of $D$ denoted by $\exp (D)$.

By a two-colored digraph $D^{(2)}$ we mean a digraph $D$ such that each of it arcs is colored by either red or blue but not both colors. An $(s, t)$-walk in $D^{(2)}$ is a walk of length $s+t$ consisting of $s$ red arcs and $t$ blue arcs. For a walk $w$ we denote $r(w)$ to be the number of red arcs in $w$ and $b(w)$ to be the number of blue arcs in $w$. The length of $w$ is $\ell(w)=r(w)+b(w)$ and the vector $\left[\begin{array}{l}r(w) \\ b(w)\end{array}\right]$ is the composition of the walk $w$. A two-colored digraph $D^{(2)}$ is primitive provided there exist nonnegative integers $h$ and $k$ such that for each pair of vertices $u$ and $v$ in $D^{(2)}$ there is an $(h, k)$-walk from $u$ to $v$. The smallest of such positive integer $h+k$ is the exponent of $D^{(2)}$ and is denoted by $\exp \left(D^{(2)}\right)$. Researches on exponents of two-colored digraphs can be found in $[2,3,5,6]$ and [7].

Let $D^{(2)}$ be a strongly connected two-colored digraph and suppose that the set of all cycles in $D^{(2)}$ is $C=\left\{C_{1}, C_{2}, \ldots, C_{q}\right\}$. We define a cycle matrix of $D^{(2)}$ 
to be a 2 by $q$ matrix

$$
M=\left[\begin{array}{llll}
r\left(C_{1}\right) & r\left(C_{2}\right) & \cdots & r\left(C_{q}\right) \\
b\left(C_{1}\right) & b\left(C_{2}\right) & \cdots & b\left(C_{q}\right)
\end{array}\right],
$$

that is $M$ is a matrix such that its $i$ th column is the composition of the $i$ th cycle $C_{i}, i=1,2, \ldots, q$. If the rank of $M$ is 1 , the content of $M$ is defined to be 0 , and the content of $M$ is defined to be the greatest common divisors of the 2 by 2 minors of $M$, otherwise. The following result, due to Fornasini and Valcher [1], gives an algebraic characterization of a primitive two-colored digraph.

Theorem 1.1. [1] Let $D^{(2)}$ be a strongly connected two-colored digraph with at least one arc of each color. Let $M$ be a cycle matrix of $D^{(2)}$. The two-colored digraph $D^{(2)}$ is primitive if and only if the content of $M$ is 1.

Let $D^{(2)}$ be a two-colored digraph on $n$ vertices $v_{1}, v_{2}, \ldots, v_{n}$. Gao and Shao [4] define a more local concept of exponents of two-colored digraphs as follows. For any vertex $v_{k}$ in $D^{(2)}, k=1,2, \ldots, n$, the exponent of the vertex $v_{k}$, denoted by $\gamma_{D^{(2)}}\left(v_{k}\right)$, is the smallest positive integer $p_{1}+p_{2}$ such that for every vertex $v$ in $D^{(2)}$ there is a $\left(p_{1}, p_{2}\right)$-walk from $v_{k}$ to $v$. It is customary to order the vertices $v_{1}, v_{2}, \ldots v_{n}$ of $D^{(2)}$ such that $\gamma_{D^{(2)}}\left(v_{1}\right) \leq \gamma_{D^{(2)}}\left(v_{2}\right) \leq \cdots \leq \gamma_{D^{(2)}}\left(v_{n}\right)$. Gao and Shao [4] discuss the vertex exponents for primitive two-colored digraphs of Wielandt type, that is a Hamiltonial digraph consisting of the cycle $v_{1} \rightarrow v_{n} \rightarrow v_{n-1} \rightarrow$ $v_{n-2} \rightarrow \cdots \rightarrow v_{2} \rightarrow v_{1}$ and the arcs $v_{1} \rightarrow v_{n-1}$. Their results show that if the twocolored Wielandt digraph $W^{(2)}$ has only one blue arc $v_{a} \rightarrow v_{a-1}, a=2,3, \ldots, n-1$, then $\gamma_{D^{(2)}}\left(v_{k}\right)=n^{2}-2 n+k-a+1$. If the two-colored Wielandt digraph has two blue arcs then $\gamma_{D^{(2)}}\left(v_{k}\right)=n^{2}-2 n+k$ or $\gamma_{D^{(2)}}\left(v_{k}\right)=n^{2}-2 n+k+1$. Suwilo [9] discusses the vertex exponents of two-colored ministrong digraphs $D^{(2)}$ on $n$ vertices whose underlying digraph is the primitive extremal ministrong digraph $D$ with $\exp (D)=n^{2}-4 n+6$.

We present formulae for vertex exponent of two-colored digraphs whose underlying digraph is the Hamiltonian digraph consisting of the cycle $v_{1} \rightarrow v_{n} \rightarrow$ $v_{n-1} \rightarrow v_{n-2} \rightarrow \cdots \rightarrow v_{2} \rightarrow v_{1}$ and the arc $v_{1} \rightarrow v_{n-2}$ where $n$ is an odd integer with $n \geq 5$. In Section 2 we discuss previous result on exponent of two-colored Hamiltonian digraph. In Section 3 we present a way in setting up a lower and an upper bound for vertex exponents. We use these results in Section 4 to find vertex exponents for the class of two-colored Hamiltonian digraphs.

\section{Two-colored Hamiltonian Digrahs}

It is a well known result, see [7], that the largest exponent of a primitive two-colored digraph lies on the interval $\left[\left(n^{3}-2 n^{2}+1\right) / 2,\left(3 n^{3}+2 n^{2}-2 n\right) / 2\right]$ when $n$ is odd and lies on the interval $\left[\left(n^{3}-5 n^{2}+7 n-2\right) / 2,\left(3 n^{3}-2 n^{2}-2 n\right) / 2\right]$ when $n$ is even. The left end of the first interval is obtained using two-colored digraphs 
consisting of two cycles whose underlying digraph is the primitive Hamiltonian digraph $L_{n}$ on $n \geq 5$ vertices which consists of an $n$-cycle $v_{1} \rightarrow v_{n} \rightarrow v_{n-1} \rightarrow v_{n-2} \rightarrow$ $v_{n-3} \rightarrow \cdots \rightarrow v_{2} \rightarrow v_{1}$ and the arc $v_{1} \rightarrow v_{n-2}$ as shown in Figure 1 . Notice that the digraph $L_{n}$ consists of exactly two cycles, they are the $n$-cycle and the $(n-2)$-cycle $v_{1} \rightarrow v_{n-2} \rightarrow v_{n-3} \rightarrow \cdots \rightarrow v_{2} \rightarrow v_{1}$. Since $L_{n}$ is primitive, it is necessary that $n$ is odd. Let $L_{n}^{(2)}$ be a two-colored digraph with underlying digraph is $L_{n}$. Let $M$ be the cycle matrix of $L_{n}^{(2)}$. By Theorem 1.1 the following lemma, see [7, 8] for proof, gives necessary and sufficient condition for $L_{n}^{(2)}$ to be a primitive two-colored digraph.

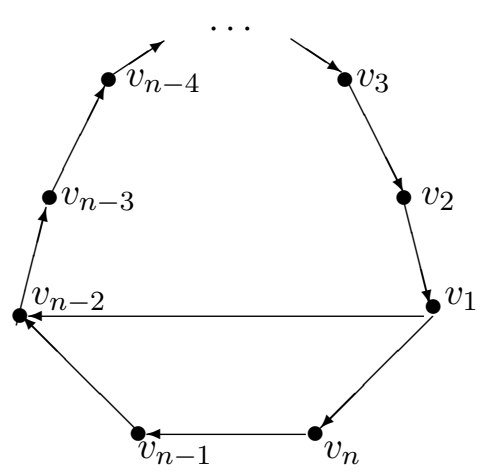

Figure 1. Digraph $L_{n}$

Lemma 2.1. $[7,8]$ Let $L_{n}^{(2)}$ be a two-colored digraph with underlying digraph $L_{n}$. The digraph $L_{n}^{(2)}$ is primitive if and only if $M=\left[\begin{array}{cc}(n-1) / 2 & (n+1) / 2 \\ (n-3) / 2 & (n-1) / 2\end{array}\right]$.

The following theorem, due Suwilo [8] see also Shader and Suwilo [7], gives the lower and upper bound for exponent of class of two-colored digraphs whose underlying digraph is the digraph $L_{n}$.

Theorem 2.2. $[7,8]$ Let $L_{n}^{(2)}$ be a two-colored digraph with underlying digraph $L_{n}$. Then $2 n^{2}-6 n+2 \leq \exp \left(L_{n}^{(2)}\right) \leq\left(n^{3}-2 n^{2}+1\right) / 2$.

Furthermore Suwilo [8] characterizes necessary and sufficient conditions for two-colored digraphs $L_{n}^{(2)}$ to have $\exp \left(L_{n}^{(2)}\right)=\left(n^{3}-2 n^{2}+1\right) / 2$ and to have $\exp \left(L_{n}^{(2)}\right)=2 n^{2}-6 n+2$, respectively.

Corollary 2.3. [8] Let $L_{n}^{(2)}$ be a two-colored digraph with underlying digraph $L_{n}$. The $\exp \left(L_{n}^{(2)}\right)=\left(n^{3}-2 n^{2}+1\right) / 2$ if and only if $L_{n}^{(2)}$ has a red path of length $(n+1) / 2$ and a blue path of length $(n-1) / 2$.

Corollary 2.4. [8] Let $L_{n}^{(2)}$ be a two-colored digraph with underlying digraph $L_{n}$. The $\exp \left(L_{n}^{(2)}\right)=2 n^{2}-6 n+2$ if and only if $L_{n}^{(2)}$ has a unique $(2,0)$-path and this path lies on both cycles. 
Lemma 2.1 implies that for a two-colored digraph $L_{n}^{(2)}$ to be primitive, the $n$-cycles must contain exactly $(n+1) / 2$ red arcs and the $(n-2)$-cycle must contain exactly $(n-1) / 2$ red arcs. Corollary 2.3 implies that for the two-colored digraph $L_{n}^{(2)}$ to have exponent $\left(n^{3}-2 n^{2}+1\right) / 2$, the $n$-cycle must contain a red path of length $(n+1) / 2$ and a blue path of length $(n-1) / 2$. This implies there are four possible two-colored digraph $L_{n}^{(2)}$ with exponent $\left(n^{3}-2 n^{2}+1\right) / 2$. We characterize them as follows.

- The two-colored digraph $L_{n}^{(2)}$ is of Type $I$ if the red arcs of $L_{n}^{(2)}$ are the arcs that lie on the path $v_{n} \rightarrow v_{n-1} \rightarrow v_{n-2} \rightarrow \cdots \rightarrow v_{(n-1) / 2}$ of length $(n+1) / 2$ plus the arc $v_{1} \rightarrow v_{n-2}$.

- The two-colored digraph $L_{n}^{(2)}$ is of Type $I I$ if the red $\operatorname{arcs}$ of $L_{n}^{(2)}$ are the arcs that lie on the path $v_{(n-1) / 2} \rightarrow v_{(n-3) / 2} \rightarrow \cdots \rightarrow 1 \rightarrow v_{n} \rightarrow v_{n-1}$ of length $(n+1) / 2$ plus the arc $v_{1} \rightarrow v_{n-2}$.

- The two-colored digraph $L_{n}^{(2)}$ is of Type $I I I$ if the red arcs of $L_{n}^{(2)}$ are the arcs that lie on the path $v_{(n+1) / 2} \rightarrow v_{(n-1) / 2} \rightarrow \cdots \rightarrow 2 \rightarrow 1 \rightarrow v_{n}$ of length $(n+1) / 2$.

- The two-colored digraph $L_{n}^{(2)}$ is of Type $I V$ if the red arcs of $L_{n}^{(2)}$ are the arcs that lie on the path $v_{n-1} \rightarrow v_{n-2} \rightarrow \cdots \rightarrow v_{(n-3) / 2}$ of length $(n+1) / 2$.

Considering Corollary 2.4, we have $\exp \left(L_{n}^{(2)}\right)=2 n^{2}-6 n+2$ if and only if the $(2,0)$-path in $L_{n}^{(2)}$ is the path $a \rightarrow a-1 \rightarrow a-2$ for some $3 \leq a \leq n-2$.

In Section 4 for two-colored digraphs $L_{n}^{(2)}$ with $\exp \left(L_{n}^{(2)}\right)=\left(n^{3}-2 n^{2}+1\right) / 2$ we show that the exponents of its vertices lie on $\left[\left(n^{3}-2 n^{2}-3 n+4\right) / 4,\left(n^{3}-2 n^{2}+\right.\right.$ $3 n+6) / 4$. For two-colored digraphs whose $\exp \left(L_{n}^{(2)}\right)=2 n^{2}-6 n+2$ we show that the exponents of its vertices lie on $\left[n^{2}-4 n+5, n^{2}-2 n-1\right]$.

\section{Bounds for vertex exponents}

In this section, a way in setting up an upper and a lower bound for vertex exponents of two-colored digraphs is discussed. We start with the lower bound of vertex exponent especially for primitive two-colored digraphs consisting of two cycles. We assume through out that the exponent of vertex $v_{k}, k=1,2, \ldots, n$ is obtained using $(s, t)$-walks.

Lemma 3.1. [9] Let $D^{(2)}$ be a primitive two-colored digraph consisting of two cycles. Let $v_{k}$ be a vertex in $D^{(2)}$ and suppose there is an $(s, t)$-walk from $v_{k}$ to each vertex $v_{j}$ in $D^{(2)}$ with $\left[\begin{array}{l}s \\ t\end{array}\right]=M\left[\begin{array}{l}q_{1} \\ q_{2}\end{array}\right]$ for some nonnegative integers $q_{1}$ and $q_{2}$. Then $\left[\begin{array}{l}q_{1} \\ q_{2}\end{array}\right] \geq M^{-1}\left[\begin{array}{l}r\left(p_{k, j}\right) \\ b\left(p_{k, j}\right)\end{array}\right]$ for some path $p_{k, j}$ from $v_{k}$ to $v_{j}$. 
Let $v_{k}$ be a vertex in $D^{(2)}$. We note that from Lemma 3.1 for the vertex $v_{k}$ and any vertex $v_{j}$ in $D$ we have

$$
\left[\begin{array}{l}
q_{1} \\
q_{2}
\end{array}\right] \geq M^{-1}\left[\begin{array}{l}
r\left(p_{k, j}\right) \\
b\left(p_{k, j}\right)
\end{array}\right]=\left[\begin{array}{l}
b\left(C_{2}\right) r\left(p_{k, j}\right)-r\left(C_{2}\right) b\left(p_{k, j}\right) \\
r\left(C_{1}\right) b\left(p_{k, j}\right)-b\left(C_{1}\right) r\left(p_{k, j}\right)
\end{array}\right] .
$$

If for some vertex $v_{j}$ we have $b\left(C_{2}\right) r\left(p_{k, j}\right)-r\left(C_{2}\right) b\left(p_{k, j}\right) \geq 0$, then we define

$$
u_{0}=b\left(C_{2}\right) r\left(p_{k, j}\right)-r\left(C_{2}\right) b\left(p_{k, j}\right)
$$

If for some vertex $v_{i}$ we have $r\left(C_{1}\right) b\left(p_{k, i}\right)-b\left(C_{1}\right) r\left(p_{k, i}\right) \geq 0$, then we define

$$
v_{0}=r\left(C_{1}\right) b\left(p_{k, i}\right)-b\left(C_{1}\right) r\left(p_{k, i}\right)
$$

By Lemma 3.1 we have $q_{1} \geq u_{0}$ and $q_{2} \geq v_{0}$. This implies

and hence

$$
\left[\begin{array}{l}
s \\
t
\end{array}\right]=M\left[\begin{array}{l}
q_{1} \\
q_{2}
\end{array}\right] \geq M\left[\begin{array}{l}
u_{0} \\
v_{0}
\end{array}\right]
$$

$$
s+t \geq\left(r\left(C_{1}\right)+b\left(C_{1}\right)\right) u_{0}+\left(r\left(C_{2}\right)+b\left(C_{2}\right)\right) v_{0}=\ell\left(C_{1}\right) u_{0}+\ell\left(C_{2}\right) v_{0} .
$$

We have proved the following theorem.

Theorem 3.2. Let $D^{(2)}$ be a primitive two-colored digraph consisting of two cycles $C_{1}$ and $C_{2}$ and let $v_{k}$ be a vertex in $D^{(2)}$. For some vertex $v_{i}$ and $v_{j}$ in $D^{(2)}$ define $u_{0}=b\left(C_{2}\right) r\left(p_{k, j}\right)-r\left(C_{2}\right) b\left(p_{k, j}\right)$ and $v_{0}=r\left(C_{1}\right) b\left(p_{k, i}\right)-b\left(C_{1}\right) r\left(p_{k, i}\right)$. Then $\left[\begin{array}{l}s \\ t\end{array}\right] \geq M\left[\begin{array}{l}u_{0} \\ v_{0}\end{array}\right]$ and hence $\gamma_{D^{(2)}}\left(v_{k}\right) \geq \ell\left(C_{1}\right) u_{0}+\ell\left(C_{2}\right) v_{0}$.

We now discuss a way in setting up an upper bound. First we consider upper bound for exponents of certain vertices two-colored digraph consisting two cycles.

Proposition 3.3. Let $D^{(2)}$ be a primitive two-colored digraph consisting of two cycles $C_{1}$ and $C_{2}$. Suppose $v_{k}$ be a vertex of $D^{(2)}$ that belongs to both cycles $C_{1}$ and $C_{2}$. If for each $i=1,2, \ldots, n$ and for some positive integers $s$ and $t$, there is a path $p_{k, i}$ from $v_{k}$ to $v_{i}$ such that the system

$$
M \mathbf{x}+\left[\begin{array}{l}
r\left(p_{k, i}\right) \\
b\left(p_{k, i}\right)
\end{array}\right]=\left[\begin{array}{l}
s \\
t
\end{array}\right]
$$

has nonnegative integer solution, then $\gamma_{D^{(2)}}\left(v_{k}\right) \leq s+t$.

Proof. Assume that the solution to the system (3) is $\mathbf{x}=\left(x_{1}, x_{2}\right)^{T}$. Since $D^{(2)}$ is primitive, then $M$ is invertible and hence $x_{1}$ and $x_{2}$ cannot be both zero. We note that $v_{k}$ belongs to both cycles and we consider three cases.

If $x_{1}, x_{2}>0$, then the walk that starts at $v_{k}$, moves $x_{1}$ and $x_{2}$ times around the cycles $C_{1}$ and $C_{2}$ respectively and back at $v_{k}$, and then moves to $v_{i}$ along the path $p_{k, i}$ is an $(s, t)$-walk from $v_{k}$ to $v_{i}$. If $x_{1}=0$ and $x_{2}>0$, then the walk that starts at $v_{k}$, moves $x_{2}$ times around the cycle $C_{2}$ and back at $v_{k}$, then moves to vertex $v_{i}$ along the path $p_{k, i}$ is an $(s, t)$-walk from $v_{k}$ to $v_{i}$. Similarly if $x_{1}>0$ and $x_{2}=0$, then then the walk that starts at $v_{k}$, moves $x_{1}$ times around the cycle $C_{1}$ 
and back at $v_{k}$, then moves to vertex $v_{i}$ along the path $p_{k, i}$ is an $(s, t)$-walk from $v_{k}$ to $v_{i}$. Therefore for every vertex $v_{i}, i=1,2, \ldots, n$ there is an $(s, t)$-walk from $v_{k}$ to $v_{i}$. The definition of exponent of vertex $v_{k}$ implies that $\gamma_{D^{(2)}}\left(v_{k}\right) \leq s+t$.

Proposition 3.4 gives an upper bound of a vertex exponent in term of the vertex exponent of a specified vertex. We define $d\left(v_{k}, v\right)$ to be the distance from $v_{k}$ to $v$, that is the length of a shortest walk from $v_{k}$ to $v$.

Proposition 3.4. [9] Let $D^{(2)}$ be a primitive two-colored digraph on $n$ vertices. Let $v$ be a vertex in $D^{(2)}$ with exponent $\gamma_{D^{(2)}}(v)$. For any vertex $v_{k}, k=1,2, \ldots, n$ in $D^{(2)}$ we have $\gamma_{D^{(2)}}\left(v_{k}\right) \leq \gamma_{D^{(2)}}(v)+d\left(v_{k}, v\right)$

\section{The vertex exponents}

In this section we discuss the vertex exponents of class of two-colored digraphs $L_{n}^{(2)}$ whose underlying digraph is the digraph $L_{n}$ in Figure 1 . We first discuss the case where $\exp \left(L_{n}^{(2)}\right)=\left(n^{3}-2 n^{2}+1\right) / 2$. Let $v_{k}$ be a vertex in $L_{n}^{(2)}$ and suppose that the red path of length $(n+1) / 2$ has $x *$ and $y *$ as its initial and terminal vertex, respectively. We use the the path $p_{k, y *}$ from $v_{k}$ to $y *$ to determine the value of $u_{0}=b\left(C_{2}\right) r\left(p_{k, y *}\right)-r\left(C_{2}\right) b\left(p_{k, y *}\right)$ in equation (1). We use the path $p_{k, x *}$ from $v_{k}$ to $x *$ in order to determine the value of $v_{0}=r\left(C_{1}\right) b\left(p_{k, x *}\right)-b\left(C_{1}\right) r\left(p_{k, x *}\right)$ in equation (2). We assume that $\gamma_{L_{n}^{(2)}}\left(v_{k}\right)$ is obtained using $(s, t)$-walks and we split our discussion into four parts depending on the type of the two-colored digraph $L_{n}^{(2)}$.

Lemma 4.1. For the two-colored digraph $L_{n}^{(2)}$ of type I we have $\gamma_{L_{n}^{(2)}}\left(v_{k}\right)=\left(n^{3}-\right.$ $\left.2 n^{2}-n+2\right) / 4+k$ for all $k=1,2, \ldots, n$.

Proof. We first show that $\gamma_{L_{n}^{(2)}}\left(v_{k}\right) \geq\left(n^{3}-2 n^{2}-n+2\right) / 4+k$ for all $k=1,2, \ldots, n$. Since the red path of length $(n+1) / 2$ in $L_{n}^{(2)}$ is the path $v_{n} \rightarrow v_{n-1} \rightarrow \cdots \rightarrow$ $v_{(n-1) / 2}$, we set $x^{*}=v_{n}$ and $y^{*}=v_{(n-1) / 2}$. We split the proof into two cases.

Case 1: $1 \leq k \leq(n-1) / 2$

Taking $y *=v_{(n-1) / 2}$, we see that there are two paths from $v_{k}$ to $v_{(n-1) / 2}$. They are an $((n+1) / 2, k)$-path and an $((n-1) / 2, k-1)$-path. Using the $((n+1) / 2, k)$-path $p_{k,(n-1) / 2}$ from $v_{k}$ to $v_{(n-1) / 2}$ and the definition of $u_{0}$ in equation (1) we find

$$
\begin{aligned}
u_{0} & =b\left(C_{2}\right) r\left(p_{k,(n-1) / 2}-r\left(C_{2}\right) b\left(p_{k,(n-1) / 2}\right)\right. \\
& =\left(\frac{n-1}{2}\right)\left(\frac{n+1}{2}\right)-\frac{n+1}{2} k=\frac{n^{2}-1}{4}-\frac{k(n+1)}{2} .
\end{aligned}
$$


Using the $((n-1) / 2, k-1)$-path $p_{k,(n-1) / 2}$ from $v_{k}$ to $v_{(n-1) / 2}$ and the definition of $u_{0}$ in equation (1) we have

$$
\begin{aligned}
u_{0} & =b\left(C_{2}\right) r\left(p_{k,(n-1) / 2}-r\left(C_{2}\right) b\left(p_{k,(n-1) / 2}\right)\right. \\
& =\left(\frac{n-1}{2}\right)\left(\frac{n-1}{2}\right)-\frac{n+1}{2}(k-1)=\frac{n^{2}+3}{4}-\frac{k(n+1)}{2} .
\end{aligned}
$$

From equations (4) and (5) we conclude that $u_{0}=\left(n^{2}-1\right) / 4-k(n+1) / 2$.

Taking $x *=v_{n}$, there is a unique path $p_{k, n}$ from $v_{k}$ to $v_{n}$ which is a $(0, k)$ path. Using this path and the definition of $v_{0}$ in equation (2) we have

$$
\begin{aligned}
v_{0} & =r\left(C_{1}\right) b\left(p_{k, n}\right)-b\left(C_{1}\right) r\left(p_{k, n}\right) \\
& =\frac{n-1}{2} k-\frac{n-3}{2}(0)=k(n-1) / 2
\end{aligned}
$$

By Theorem 3.2 we have

$$
\begin{aligned}
{\left[\begin{array}{l}
s \\
t
\end{array}\right] \geq M } & {\left[\begin{array}{l}
u_{0} \\
v_{0}
\end{array}\right]=M\left[\begin{array}{l}
\left(n^{2}-1\right) / 4-k(n+1) / 2 \\
k(n-1) / 2
\end{array}\right] } \\
& =\left[\begin{array}{l}
\left(n^{3}-n^{2}-n+1\right) / 8 \\
\left(n^{3}-3 n^{2}-n+3+8 k\right) / 8
\end{array}\right] .
\end{aligned}
$$

Therefore, we conclude that

$$
\gamma_{L_{n}^{(2)}}\left(v_{k}\right)=s+t \geq\left(n^{3}-2 n^{2}-n+2\right) / 4+k
$$

for all $k=1,2, \ldots,(n-1) / 2$.

Case 2: $(n+1) / 2 \leq k \leq n$

Taking $y^{*}=v_{(n-1) / 2}$, then there is a unique path $p_{k,(n-1) / 2}$ from $v_{k}$ to $v_{(n-1) / 2}$ which is a $(k-(n-1) / 2,0)$-path. Using this path and considering the definition of $u_{0}$ in equation (1) we have $u_{0}=k(n-1) / 2-(n-1)^{2} / 4$. Taking $x^{*}=v_{n}$, there is a unique path $p_{k, n}$ from $v_{k}$ to $v_{n}$. This path is a $(k-(n-1) / 2,(n-1) / 2)$-path. Using this path and the definition of $v_{0}$ in equation (2) we get $v_{0}=(n-1)(2 n-$ $4) / 4-k(n-3) / 2$. By Theorem 3.2 we get

$$
\gamma_{L_{n}^{(2)}}\left(v_{k}\right) \geq \ell\left(C_{1}\right) u_{0}+\ell\left(C_{2}\right) v_{0}=\left(n^{3}-2 n^{2}-n+2\right) / 4+k
$$

for all $k=(n+1) / 2,(n+3) / 2, \ldots, n$.

Combining (7) and (8) we conclude that

$$
\gamma_{L_{n}^{(2)}}\left(v_{k}\right) \geq\left(n^{3}-2 n^{2}-n+2\right) / 4+k .
$$

for all $k=1,2, \ldots, n$.

We next show the upper bound, that is $\gamma_{L_{n}^{(2)}}\left(v_{k}\right) \leq\left(n^{3}-2 n^{2}-n+2\right) / 4+k$ for all $k=1,2, \ldots, n$. We first show that $\gamma_{L_{n}^{(2)}}\left(v_{1}\right)=\left(n^{3}-2 n^{2}-n+2\right) / 4+1$ and then we use Proposition 3.4 in order to determine the upper bound for exponents of other vertices. From (9) it is known that $\gamma_{L_{n}^{(2)}}\left(v_{1}\right) \geq\left(n^{3}-2 n^{2}-n+2\right) / 4+1$. Thus, it remains to show that $\gamma_{L_{n}^{(2)}}\left(v_{1}\right) \leq\left(n^{3}-2 n^{2}-n+2\right) / 4+1$. By considering 
equation (6) we show that for each $i=1,2, \ldots, n$ there is a walk from $v_{1}$ to $v_{i}$ with composition

$$
\left[\begin{array}{l}
s \\
t
\end{array}\right]=\left[\begin{array}{c}
\left(n^{3}-n^{2}-n+1\right) / 8 \\
\left(n^{3}-3 n^{2}-n+11\right) / 8
\end{array}\right] .
$$

Let $p_{1, i}$ be a path from $v_{1}$ to $v_{i}, i=1,2, \ldots, n$. Notice that since $M$ is an invertible matrix, the system

$$
M\left[\begin{array}{l}
x_{1} \\
x_{2}
\end{array}\right]+\left[\begin{array}{l}
r\left(p_{1 i}\right) \\
b\left(p_{1 i}\right)
\end{array}\right]=\left[\begin{array}{l}
\left(n^{3}-n^{2}-n+1\right) / 8 \\
\left(n^{3}-3 n^{2}-n+11\right) / 8
\end{array}\right]
$$

has solution the integer vector

$$
\left[\begin{array}{l}
x_{1} \\
x_{2}
\end{array}\right]=\left[\begin{array}{l}
(n-3)(n+1) / 4+(n+1) b\left(p_{1 i}\right) / 2-(n-1) r\left(p_{1 i}\right) / 2 \\
(n-1) / 2+(n-3) r\left(p_{1 i}\right) / 2-(n-1) b\left(p_{1 i}\right) / 2
\end{array}\right] .
$$

If $i=1$, we can choose $r\left(p_{1,1}\right)=b\left(p_{1,1}\right)=0$ and hence we have that $x_{1}=$ $\left(n^{2}-2 n-3\right) / 4>0$ and $x_{2}=(n-1) / 2>0$. If $i=n$, then using the $(0,1)$-path we have $x_{1}=(n-3)(n+1) / 4+(n+1) / 2 \geq 0$ and $x_{2}=0$. If $i=(n-1) / 2$, then using the $((\mathrm{n}+1) / 2,1)$-path $v_{1} \rightarrow v_{n} \rightarrow v_{n-1} \rightarrow \cdots \rightarrow v_{(n-1) / 2}$ we have $x_{1}=0$ and $x_{2}=(n+1)(n-3) / 4$. Notice that for each vertex $v_{i}, i \neq n,(n-1) / 2$, there exists a path $p_{1 i}$ from $v_{1}$ to $v_{i}$ with $0 \leq r\left(p_{1 i}\right) \leq(n-3) / 2$ and $0 \leq b\left(p_{1 i}\right) \leq(n-3) / 2$. More over if $b\left(p_{1 i}\right) \geq 1$, then either $r\left(p_{1 i}\right)=(n-1) / 2$ or $r\left(p_{1 i}\right)=1$. These facts imply that $x_{1}>0$ and $x_{2}>0$. Hence we now conclude that the system (11) has a nonnegative integer solution. Proposition 3.3 implies that $\gamma_{L_{n}^{(2)}}\left(v_{1}\right) \leq\left(n^{3}-2 n^{2}-n+2\right) / 4+1$. Combining this with (9) we have $\gamma_{L_{n}^{(2)}}\left(v_{1}\right)=\left(n^{3}-2 n^{2}-n+2\right) / 4+1$. Since for every $k=2,3, \ldots, n$ we have $d\left(v_{k}, v_{1}\right)=k-1$, Proposition 3.4 implies that

$$
\gamma_{L_{n}^{(2)}}\left(v_{k}\right) \leq\left(n^{3}-2 n^{2}-n+2\right) / 4+k .
$$

for all $k=1,2, \ldots, n$.

Now using (9) and (12) we conclude that $\gamma_{L_{n}^{(2)}}\left(v_{k}\right)=\left(n^{3}-2 n^{2}-n+2\right) / 4+k$ for all $k=1,2, \ldots, n$.

Lemma 4.2. For the two-colored digraph $L_{n}^{(2)}$ of type II we have $\gamma_{L_{n}^{(2)}}\left(v_{k}\right)=\left(n^{3}-\right.$ $\left.2 n^{2}-3 n+4\right) / 4+k$ for all $k=1,2, \ldots, n$.

Proof. We first show that $\gamma_{L_{n}^{(2)}}\left(v_{k}\right) \geq\left(n^{3}-2 n^{2}-3 n+4\right) / 4+k$ for all $k=$ $1,2, \ldots, n$. Since the red path of length $(n+1) / 2$ in $L_{n}^{(2)}$ is the path $v_{(n-1) / 2} \rightarrow$ $v_{(n-3) / 2} \rightarrow \cdots \rightarrow v_{1} \rightarrow v_{n} \rightarrow v_{n-1}$, we set $x^{*}=v_{(n-1) / 2}$ and $y^{*}=v_{n-1}$. We split the proof into three cases.

Case 1: $1 \leq k \leq(n-1) / 2$

Taking $y^{*}=v_{n-1}$, then there is a unique path $p_{k, n-1}$ from $v_{k}$ to $v_{n-1}$. This path is a $(k+1,0)$-path. Using this path and the definition of $u_{0}$ in equation (1) we have

$$
u_{0}=(k+1)(n-1) / 2
$$


Taking $x^{*}=v_{(n-1) / 2}$, there are two paths $p_{k,(n-1) / 2}$ from $v_{k}$ to $v_{(n-1) / 2}$. They are a $(k,(n-3) / 2)$-path and a $(k+1,(n-1) / 2)$-path. Using the $(k,(n-3) / 2)$ path and the definition of $v_{0}$ in equation (2) we have $v_{0}=(n-3)(n-1-2 k) / 4$. Using the $(k+1,(n-1) / 2)$-path and the definition of $v_{0}$ in equation $(2)$ we have $v_{0}=(n-3)(n-1-2 k) / 4+1$. Hence, we conclude that

$$
v_{0}=(n-3)(n-1-2 k) / 4 .
$$

By Theorem 3.2, equations (13) and (14) we conclude that

$$
\left[\begin{array}{l}
s \\
t
\end{array}\right] \geq M\left[\begin{array}{l}
(k+1)(n-1) / 2 \\
(n-3)(n-1-2 k) / 4
\end{array}\right]=\left[\begin{array}{l}
\left(n^{3}-n^{2}-5 n+5\right) / 8+k \\
\left(n^{3}-3 n^{2}-n+3\right) / 8
\end{array}\right] .
$$

From (15) we conclude that

$$
s+t=\gamma_{L_{n}^{(2)}}\left(v_{k}\right) \geq\left(n^{3}-2 n^{2}-3 n+4\right) / 4+k
$$

for all $k=1,2, \ldots,(n-1) / 2$.

Case 2: $(n+1) / 2 \leq k \leq n-1$

Taking $y^{*}=v_{n-1}$, there is a unique path $p_{k, n-1}$ from $v_{k}$ to $v_{n-1}$ which is a $((n+$ $1) / 2, k-(n-1) / 2)$-path. Using this path and the definition of $u_{0}$ in equation (1) we have

$$
u_{0}=\left(n^{2}-1\right) / 2-k(n+1) / 2 .
$$

Taking $x^{*}=v_{(n-1) / 2}$, there is a unique path $p_{k,(n-1) / 2}$ from $v_{k}$ to $v_{(n-1) / 2}$. This path is a $(0, k-(n-1) / 2)$-path. Using this path and the definition of $v_{0}$ in equation (2), we have

$$
v_{0}=k(n-1) / 2-(n-1)^{2} / 4 .
$$

Equations (17), (18) and Theorem 3.2 imply that

$$
\gamma_{L_{n}^{(2)}}\left(v_{k}\right) \geq \ell\left(C_{1}\right) u_{0}+\ell\left(C_{2}\right) v_{0}=\left(n^{3}-2 n^{2}-3 n+4\right) / 4+k
$$

for all $k=(n+1) / 2,(n+3) / 2, \ldots, n-1$.

Case 3: $k=n$

There is a $(1,0)$-path $p_{k, n-1}$ from $v_{k}$ to $v_{n-1}$. Using this path and the definition of $u_{0}$ in equation (1) we have $u_{0}=(n-1) / 2$. There is a $(1,(n-1) / 2)$-path $p_{k,(n-1) / 2}$ from $v_{k}$ to $v_{(n-1) / 2}$. Using this path and the definition of $v_{0}$ in equation (2) we find that $v_{0}=(n-1)^{2} / 4-(n-3) / 2$. Theorem 3.2 implies that

$$
\begin{aligned}
\gamma_{L_{n}^{(2)}}\left(v_{k}\right) & \geq \ell\left(C_{1}\right) u_{0}+\ell\left(C_{2}\right) v_{0}=\left(n^{3}-2 n^{2}-3 n+4\right) / 4+n \\
& =\left(n^{3}-2 n^{2}-3 n+4\right) / 4+k
\end{aligned}
$$

for $k=n$.

Now from (16), (19), and (20) we conclude that

$$
\gamma_{L_{n}^{(2)}}\left(v_{k}\right) \geq\left(n^{3}-2 n^{2}-3 n+4\right) / 4+k
$$

for all $k=1,2, \ldots, n$. 
We next show the upper bound, that is $\gamma_{L_{n}^{(2)}}\left(v_{k}\right) \leq\left(n^{3}-2 n^{2}-3 n+4\right) / 4+k$ for all $k=1,2, \ldots, n$. We first show that $\gamma_{L_{n}^{(2)}}\left(v_{1}\right)=\left(n^{3}-2 n^{2}-3 n+4\right) / 4+1$ and then we use Proposition 3.4 in order to determine the upper bound for exponents of other vertices. From (16) it is known that $\gamma_{L_{n}^{(2)}}\left(v_{1}\right) \geq\left(n^{3}-2 n^{2}-3 n+4\right) / 4+1$. Thus, it remains to show that $\gamma_{L_{n}^{(2)}}\left(v_{1}\right) \leq\left(n^{3}-2 n^{2}-3 n+4\right) / 4+1$. By considering (16) we show that for each $i=1,2, \ldots, n$ there is a walk from $v_{1}$ to $v_{i}$ consisting of $\left(n^{3}-n^{2}-5 n+13\right) / 8$ red arcs and $\left(n^{3}-3 n^{2}-n+3\right) / 8$ blue arcs.

Let $p_{1 i}$ be a path form $v_{1}$ to $v_{i}, i=1,2, \ldots, n$. Notice that the system

$$
M\left[\begin{array}{l}
x_{1} \\
x_{2}
\end{array}\right]+\left[\begin{array}{l}
r\left(p_{1 i}\right) \\
b\left(p_{1 i}\right)
\end{array}\right]=\left[\begin{array}{l}
\left(n^{3}-n^{2}-5 n+13\right) / 8 \\
\left(n^{3}-3 n^{2}-n+3\right) / 8
\end{array}\right]
$$

has integer solution

$$
\left[\begin{array}{l}
x_{1} \\
x_{2}
\end{array}\right]=\left[\begin{array}{l}
b\left(p_{1, i}\right)+\left(2+b\left(p_{1, i}\right)-r\left(p_{1, i}\right)\right)(n-1) / 2 \\
(n-3)\left[n-3+2 r\left(p_{1, i}\right)\right] / 4-b\left(p_{1, i}\right)(n-1) / 2
\end{array}\right] .
$$

If $i=1$, we choose $r\left(p_{1,1}\right)=b\left(p_{1,1}\right)=0$. This implies $x_{1}=n-1>0$ and $x_{2}=(n-3)^{2} / 4>0$. Since for every $i=2,3 \ldots, n$ there is a path $p_{1 i}$ from $v_{1}$ to $v_{i}$ with $b\left(p_{1 i}\right)-r\left(p_{1 i}\right) \geq-1$, we have $x_{1}=b\left(p_{1, i}\right)+\left[2+b\left(p_{1, i}\right)-r\left(p_{1, i}\right)\right](n-1) / 2 \geq 0$. Notice also that for any $i=2,3, \ldots, n$ there is a path $p_{1 i}$ from $v_{1}$ to $v_{i}$ with $b\left(p_{1 i}\right) \leq(n-3) / 2$ and every such path $p_{1 i}$ has $r\left(p_{1 i}\right) \geq 1$. Hence $x_{2}=(n-3)[n-$ $\left.3+2 r\left(p_{1, i}\right)\right] / 4-b\left(p_{1, i}\right)(n-1) / 2 \geq 0$. Therefore the system (22) has a nonnegative integer solution. Since $v_{1}$ lies on both cycles, Proposition 3.3 implies that

$$
\gamma_{L_{n}^{(2)}}\left(v_{1}\right) \leq\left(n^{3}-2 n^{2}-3 n+4\right) / 4+1 .
$$

Now combining (16) and (23) we find that $\gamma_{L_{n}^{(2)}}\left(v_{1}\right)=\left(n^{3}-2 n^{2}-3 n+4\right) / 4+1$. Since for any $k=1,2, \ldots, n$ we have $d\left(v_{k}, v_{1}\right)=k-1$, by Proposition 3.4 we have

$$
\gamma_{L_{n}^{(2)}}\left(v_{k}\right) \leq\left(n^{3}-2 n^{2}-3 n+4\right) / 4+k
$$

for all $k=1,2, \ldots, n$.

Finally, combining (21) and (24) we conclude that $\gamma_{L_{n}^{(2)}}\left(v_{k}\right)=\left(n^{3}-2 n^{2}-\right.$ $3 n+4) / 4+k$ for all $k=1,2, \ldots, n$.

Lemma 4.3. For the two-colored digraph $L_{n}^{(2)}$ of type III we have $\gamma_{L_{n}^{(2)}}\left(v_{k}\right)=$ $\left(n^{3}-2 n^{2}-3 n\right) / 4+k$ for all $k=1,2, \ldots, n$.

Proof. We first show that $\gamma_{L_{n}^{(2)}}\left(v_{k}\right) \geq\left(n^{3}-2 n^{2}-3 n\right) / 4+k$ for all $k=1,2, \ldots, n$. Since the red path of length $(n+1) / 2$ is the path $v_{(n+1) / 2} \rightarrow v_{(n-1) / 2} \rightarrow \cdots \rightarrow$ $v_{1} \rightarrow v_{n}$, we set $x^{*}=v_{(n+1) / 2}$ and $y^{*}=v_{n}$. We split the proof into two cases.

Case 1: $1 \leq k \leq(n+1) / 2$

Considering the $(k, 0)$-path from $v_{k}$ to $v_{n}$ and the definition of $u_{0}$ in equation (1) we have

$$
u_{0}=k(n-1) / 2 .
$$


We note that there are two paths from $v_{k}$ to $v_{(n+1) / 2}$. They are a $(k-$ $1,(n-3) / 2)$-path and a $(k,(n-1) / 2)$-path. Using the $(k-1,(n-3) / 2)$-path and the definition of $v_{0}$ in equation $(2)$ we have that $v_{0}=(n-3)(n-2 k+1) / 4$. Using the $(k,(n-1) / 2)$-path and the definition of $v_{0}$ in equation $(2)$ we have $v_{0}=(n-3)(n-2 k+1) / 4+1$. Hence, we conclude that

$$
v_{0}=(n-3)(n-2 k+1) / 4 .
$$

Now by considering Theorem 3.2, equation (25) and equation (26) we have

$$
\left[\begin{array}{l}
s \\
t
\end{array}\right] \geq M\left[\begin{array}{l}
(n-1) / 2 \\
(n-3)(n-2 k+1) / 4
\end{array}\right]=\left[\begin{array}{l}
\left(n^{3}-n^{2}-5 n-3\right) / 8+k \\
\left(n^{3}-3 n^{2}-n+3\right) / 8
\end{array}\right] .
$$

Thus from (27) we conclude that

$$
\gamma_{L_{n}^{(2)}}\left(v_{k}\right) \geq\left(n^{3}-2 n^{2}-3 n\right) / 4+k
$$

for all $k=1,2, \ldots,(n+1) / 2$.

Case 2: $(n+3) / 2 \leq k \leq n$

Considering the $((n+1) / 2, k-(n+1) / 2)$-path from $v_{k}$ to $v_{n}$ and the definition of $u_{0}$ in equation (1) we have $u_{0}=(n+1)(n-k) / 2$. Considering the $(0, k-(n+1) / 2)$ path from $v_{k}$ to $v_{(n+1) / 2}$ and the definition of $v_{0}$ in equation (2) we have $v_{0}=$ $(n-1)(2 k-n-1) / 4$. By Theorem 3.2, we have

$$
\gamma_{L_{n}^{(2)}}\left(v_{k}\right) \geq \ell\left(C_{1}\right) u_{0}+\ell\left(C_{2}\right) v_{0}=\left(n^{2}-2 n^{2}-3 n\right) / 4+k
$$

for all $k=(n+3) / 2,(n+5) / 2, \ldots, n$.

Now from (28) and (29) we conclude that

$$
\gamma_{L_{n}^{(2)}}\left(v_{k}\right) \geq\left(n^{3}-2 n^{2}-3 n\right) / 4+k
$$

for all $k=1,2, \ldots, n$.

We next show that $\gamma_{L_{n}^{(2)}}\left(v_{k}\right) \leq\left(n^{3}-2 n^{2}-3 n\right) / 4+k$ for all $k=1,2, \ldots, n$. For this purpose we first show that $\gamma_{L_{n}^{(2)}}\left(v_{1}\right)=\left(n^{3}-2 n^{2}-3 n\right) / 4+1$ and then we use Proposition 3.4 in order to get the upper bounds for $\gamma_{L_{n}^{(2)}}\left(v_{k}\right)$ for $k=2,3, \ldots, n$. From (30) it is inferred that $\gamma_{L_{n}^{(2)}}\left(v_{1}\right) \geq\left(n^{3}-2 n^{2}-3 n\right) / 4+1$. It remains to show that $\gamma_{L_{n}^{(2)}}\left(v_{1}\right) \leq\left(n^{3}-2 n^{2}-3 n\right) / 4+1$.

By considering (27) we show that for each vertex $v_{i}, i=1,2, \ldots, n$, there is a walk from $v_{1}$ to $v_{i}$ consisting of $\left(n^{3}-n^{2}-5 n+5\right) / 8$ red $\operatorname{arcs}$ and $\left(n^{3}-3 n^{2}-n+3\right) / 8$ blue arcs. For $i=1,2, \ldots, n$ let $p_{1, i}$ be a path from $v_{1}$ to $v_{i}$. Consider the system of equations

$$
M\left[\begin{array}{l}
x_{1} \\
x_{2}
\end{array}\right]+\left[\begin{array}{l}
r\left(p_{1, i}\right) \\
b\left(p_{1, i}\right)
\end{array}\right]=\left[\begin{array}{c}
\left(n^{3}-n^{2}-5 n+5\right) / 8 \\
\left(n^{3}-3 n^{2}-n+3\right) / 8
\end{array}\right] .
$$

The solution to the system (31) is the integer vector

$$
\left[\begin{array}{l}
x_{1} \\
x_{2}
\end{array}\right]=\left[\begin{array}{l}
b\left(p_{1, i}\right)+\left(1+b\left(p_{1, i}\right)-r\left(p_{1, i}\right)\right)(n-1) / 2 \\
(n-3)(n-1) / 4+r\left(p_{1, i}\right)(n-3) / 2-b\left(p_{1, i}\right)(n-1) / 2
\end{array}\right] .
$$


If $i=1$, we can choose $r\left(p_{1,1}\right)=b\left(p_{1,1}\right)=0$. This implies $x_{1}=(n-1) / 2>0$ and $x_{2}=\left(n^{2}-4 n+3\right) / 4>0$. Notice that for each $i=2,3, \ldots, n$ there is a path $p_{1 i}$ from $v_{1}$ to $v_{i}$ with $b\left(p_{1 i}\right) \leq(n-3) / 2$. Hence, $x_{2} \geq 0$. Moreover, there is a path from $v_{1}$ to $v_{i}$ with $1+b\left(p_{1 i}\right)-r\left(p_{i 1}\right) \geq 0$. Hence we have $x_{1} \geq 0$. These imply that the system (31) has a nonnegative integer solution. Since the vertex $v_{1}$ lies on both cycles, by Proposition 3.3 we conclude that $\gamma_{L_{n}^{(2)}}\left(v_{1}\right) \leq\left(n^{3}-2 n^{2}-3 n\right) / 4+1$. Since for each vertex $v_{k}, k=2,3, \ldots, n, d\left(v_{k}, v_{1}\right)=k-1$, Proposition 3.4 guarantees that

$$
\gamma_{L_{n}^{(2)}}\left(v_{k}\right) \leq\left(n^{3}-2 n^{2}-3 n\right) / 4+k
$$

for all $k=1,2, \ldots, n$.

Now combining (30) and (32) we conclude that $\gamma_{L_{n}^{(2)}}\left(v_{k}\right)=\left(n^{3}-2 n^{2}-3 n\right) / 4+$ $k$ for all $k=1,2, \ldots, n$.

Lemma 4.4. For the two-colored digraph $L_{n}^{(2)}$ of type $I V$ we have $\gamma_{L_{n}^{(2)}}\left(v_{k}\right)=$ $\left(n^{3}-2 n^{2}-n+6\right) / 4+k$ for all $k=1,2, \ldots, n$.

Proof. Since the red path of length $(n+1) / 2$ is the path $v_{n-1} \rightarrow v_{n-2} \rightarrow \cdots \rightarrow$ $v_{(n-3) / 2}$, we set $x^{*}=v_{n-1}$ and $y^{*}=v_{(n-3) / 2}$. We split the proof into three cases depending on the position of $v_{k}$.

Case $1: 1 \leq k \leq(n-3) / 2$

There are two paths from $v_{k}$ to $v_{(n-3) / 2}$. They are a $((n-1) / 2, k)$-path and a $((n+1) / 2, k+1)$-path. Using the $((n-1) / 2, k)$-path we find from the definition of $u_{0}$ in equation (1) that $u_{0}=(n-1)^{2} / 4-k(n+1) / 2$. Using the $((n+1) / 2, k+1)$-path we find from the definition of $u_{0}$ in equation (1) that $u_{0}=(n-1)^{2} / 4-k(n+1) / 2-1$. Hence we choose

$$
u_{0}=(n-1)^{2} / 4-k(n+1) / 2-1=\left(n^{2}-1\right) / 4-(k+1)(n+1) / 2 .
$$

We note that there is a unique path $p_{k, n-1}$ from $v_{k}$ to $v_{n-1}$ which is a $(0, k+1)$ path. Using this path we find from the definition of $v_{0}$ in equation (2) that

$$
v_{0}=(k+1)(n-1) / 2
$$

Theorem 3.2, equation (33) and equation (34) imply that

$$
\left[\begin{array}{l}
s \\
t
\end{array}\right] \geq M\left[\begin{array}{l}
u_{0} \\
v_{0}
\end{array}\right]=\left[\begin{array}{l}
\left(n^{3}-n^{2}-n+1\right) / 8 \\
\left(n^{3}-3 n^{2}-n+11\right) / 8+k
\end{array}\right] .
$$

From (35) we conclude that

$$
\gamma_{L_{n}^{(2)}}\left(v_{k}\right) \geq\left(n^{3}-2 n^{2}-n+6\right) / 4+k
$$

for all $k=1,2, \ldots,(n-3) / 2$.

Case $2:(n-1) / 2 \leq k \leq n-1$

There is a unique $p_{k,(n-3) / 2}$-path from $v_{k}$ to $v_{(n-3) / 2}$ which is a $(k-(n-3) / 2,0)$ path. Using this path, from the definition of $u_{0}$ in equation (1) we find that $u_{0}=k(n-1) / 2-(n-1)(n-3) / 4$. There is a unique path $p_{k, n-1}$ from $v_{k}$ to $v_{n-1}$ 
which is a $(k-(n-3) / 2,(n-1) / 2)$-path. Using this path, from the definition of $v_{0}$ in equation (2) we find that $v_{0}=(n-1)^{2} / 4+(n-3)^{2} / 4-k(n-3) / 2$. Theorem 3.2 implies

$$
\begin{aligned}
\gamma_{L_{n}^{(2)}}\left(v_{k}\right) \geq & \ell\left(C_{1}\right) u_{0}+\ell\left(C_{2}\right) v_{0} \\
= & (n-2)(k(n-1) / 2-(n-1)(n-3) / 4) \\
& +n\left[(n-1)^{2} / 4+(n-3)^{2} / 4-k(n-3) / 2\right] \\
= & \left(n^{3}-2 n^{2}-n+6\right) / 4+k
\end{aligned}
$$

for all $k=(n-1) / 2,(n+1) / 2, \ldots, n-1$.

Case 3: $k=n$

There is a unique path from $v_{k}$ to $v_{(n-1) / 2}$ which is a $((n+1) / 2,1)$-path. Using this path we find from the definition of $u_{0}$ in equation (1) that $u_{0}=\left(n^{2}-1\right) / 4-(n+1) / 2$. There is a unique path $p_{k, n-1}$ from $v_{k}$ to $v_{n-1}$ which is a $(0,1)$-path. Using this path we find from the definition of $v_{0}$ in equation $(2)$ that $v_{0}=(n-1) / 2$. Theorem 3.2 implies

$$
\left[\begin{array}{l}
s \\
t
\end{array}\right] \geq M\left[\begin{array}{l}
u_{0} \\
v_{0}
\end{array}\right]=\left[\begin{array}{l}
\left(n^{3}-n^{2}-n+1\right) / 8 \\
\left(n^{3}-3 n^{2}-n+11\right) / 8
\end{array}\right],
$$

and hence $\gamma_{L_{n}^{(2)}}\left(v_{k}\right) \geq\left(n^{3}-2 n^{2}-5 n+6\right) / 4+n$. We note that for the $(0,1)$-path from $v_{n}$ to $v_{n-1}$, the system

$$
M\left[\begin{array}{l}
x_{1} \\
x_{2}
\end{array}\right]+\left[\begin{array}{l}
0 \\
1
\end{array}\right]=\left[\begin{array}{l}
\left(n^{3}-n^{2}-n+1\right) / 8 \\
\left(n^{3}-3 n^{2}-n+11\right) / 8
\end{array}\right]
$$

has nonnegative integer solution $x_{1}=\left(n^{2}-1\right) / 4$ and $x_{2}=0$. This implies there is no walk from $v_{n}$ to $v_{n-1}$ with composition $\left[\begin{array}{c}\left(n^{3}-n^{2}-n+1\right) / 8 \\ \left(n^{3}-3 n^{2}-n+11\right) / 8\end{array}\right]$. Hence $\gamma_{L_{n}^{(2)}}\left(v_{n}\right)>\left(n^{3}-2 n^{2}-5 n+6\right) / 4+n$. Notice that the shortest walk from $v_{n}$ to $v_{n-1}$ with at least $\left(n^{3}-n^{2}-n+1\right) / 8$ red arcs and $\left(n^{3}-3 n^{2}-n+11\right) / 8$ blue arcs is the walk that starts at $v_{n}$, moves to $v_{n-2}$ and then moves $\left(n^{2}-1\right) / 4$ times around the cycle $C_{1}$ and back at $v_{n-2}$, finally moves to $v_{n-1}$. The composition of this walk is $\left[\begin{array}{l}\left(n^{3}-n^{2}+3 n+5\right) / 8 \\ \left(n^{3}-3 n^{2}+3 n+7\right) / 8\end{array}\right]$. Thus we now have

$$
\gamma_{L_{n}^{(2)}}\left(v_{n}\right) \geq\left(n^{3}-2 n^{2}+3 n+6\right) / 4=\left(n^{3}-2 n^{2}-n+6\right) / 4+n .
$$

From (36), (37) and (38) we conclude that

$$
\gamma_{L_{n}^{(2)}}\left(v_{k}\right) \geq\left(n^{3}-2 n^{2}-n+6\right) / 4+k
$$

for all $k=1,2, \ldots, n$.

We next show $\gamma_{L_{n}^{(2)}}\left(v_{k}\right) \leq\left(n^{3}-2 n^{2}-n+6\right)+k$ by first showing that $\gamma_{L_{n}^{(2)}}\left(v_{1}\right)=\left(n^{3}-2 n^{2}-n+6\right) / 4+1$, and then use Proposition 3.4 to get upper bound for exponent of the vertex $v_{k}, k=2,3, \ldots, n$. From (36) we know that for $k=1, \gamma_{L_{n}^{(2)}}\left(v_{1}\right) \geq\left(n^{3}-2 n^{2}-n+6\right) / 4+1$ and from (35) we know that this bound 
is obtained by walks with composition $\left[\begin{array}{l}\left(n^{3}-n^{2}-n+1\right) / 8 \\ \left(n^{3}-3 n^{2}-n+19\right) / 8\end{array}\right]$. It remains to show that $\gamma_{L_{n}^{(2)}}\left(v_{1}\right) \leq\left(n^{3}-2 n^{2}-n+6\right) / 4+1$. For each $i=1,2, \ldots, k$ we show that there is an $(s, t)$-walk $w_{1, i}$ from $v_{1}$ to $v_{i}$ with composition

$$
\left[\begin{array}{l}
r\left(w_{1, i}\right) \\
b\left(w_{1, i}\right)
\end{array}\right]=\left[\begin{array}{l}
\left(n^{3}-n^{2}-n+1\right) / 8 \\
\left(n^{3}-3 n^{2}-n+19\right) / 8
\end{array}\right] .
$$

For any vertex $v_{i}, i=1,2,3, \ldots, n$ let $p_{1 i}$ be a path from $v_{1}$ to $v_{i}$. The system

$$
M\left[\begin{array}{l}
x_{1} \\
x_{2}
\end{array}\right]+\left[\begin{array}{l}
r\left(p_{1 i}\right) \\
b\left(p_{1 i}\right)
\end{array}\right]=\left[\begin{array}{l}
\left(n^{3}-n^{2}-n+1\right) / 8 \\
\left(n^{3}-3 n^{2}-n+19\right) / 8
\end{array}\right]
$$

has integer solution

$$
\left[\begin{array}{l}
x_{1} \\
x_{2}
\end{array}\right]=\left[\begin{array}{l}
r\left(p_{1, i}\right)+\left((n-5) / 2+b\left(p_{1, i}\right)-r\left(p_{1, i}\right)\right)(n+1) / 2 \\
\left(2-b\left(p_{1, i}\right)\right)(n-1) / 2+r\left(p_{1, i}\right)(n-3) / 2
\end{array}\right] .
$$

If $i=1$, we can choose $r\left(p_{1,1}\right)=b\left(p_{1,1}\right)=0$. This implies $x_{1}=\left(n^{2}-4 n-5\right) / 4>0$ and $x_{2}=n-1>0$. We note that for any vertex $v_{i}, i=2,3, \ldots, n$ there is a path $p_{1 i}$ from $v_{1}$ to $v_{i}$ with $2 \leq b\left(p_{1 i}\right) \leq(n-3) / 2$. Moreover, if $3 \leq b\left(p_{1 i}\right) \leq(n-3) / 2$, then $r\left(p_{1 i}\right)=(n-1) / 2$. Hence $x_{2} \geq 0$. Notice also that for any vertex $v_{i}, i=2,3, \ldots, n$ we can find a path $p_{1 i}$ with $b\left(p_{1 i}\right)-r\left(p_{1 i}\right) \geq-(n-5) / 2$. Hence $x_{1} \geq 0$. Hence the system (40) has a nonnegative integer solution. Since the vertex $v_{1}$ lies on both cycles, Proposition 3.3 guarantees that $\gamma_{L_{n}^{(2)}}\left(v_{1}\right) \leq\left(n^{3}-2 n^{2}-n+6\right) / 4+1$. By considering equation (39) we conclude that $\gamma_{L_{n}^{(2)}}\left(v_{1}\right)=\left(n^{3}-2 n^{2}-n+6\right) / 4+1$. Since for each $k=2,3, \ldots, n$ we have $d\left(v_{k}, v_{1}\right)=k-1$, Proposition 3.4 implies that

$$
\gamma_{L_{n}^{(2)}}\left(v_{k}\right) \leq\left(n^{3}-2 n^{2}-n+6\right) / 4+k
$$

for $k=1,2, \ldots, n$.

Combining (39) and (41) we conclude that $\gamma\left(v_{k}\right)=\left(n^{3}-2 n^{2}-n+6\right)+k$ for all $k=1,2, \ldots, n$.

Theorem 4.5. Let $L_{n}^{(2)}$ be a primitive two-colored digraph on $n \geq 5$ vertices whose underlying digraph is the digraph $L_{n}$ in Figure 1 . If $\exp \left(L_{n}^{(2)}\right)=\left(n^{3}-2 n^{2}+1\right) / 2$, then $\left(n^{3}-2 n^{2}-3 n+4\right) / 4 \leq \gamma_{L_{n}^{(2)}}\left(v_{k}\right) \leq\left(n^{3}-2 n^{2}+3 n+6\right) / 4$ for all $k=1,2, \ldots, n$

Proof. By Lemma 4.1 through Lemma 4.4 for each $k=1,2, \ldots, n$ we have that $\left(n^{3}-2 n^{2}-3 n\right) / 4+k \leq \gamma_{L_{n}^{(2)}}\left(v_{k}\right) \leq\left(n^{3}-2 n^{2}-n+6\right) / 4+k$. This implies for any $k=1,2, \ldots, n$ we have $\left(n^{3}-2 n^{2}-3 n+4\right) / 4 \leq \gamma_{L_{n}^{(2)}}\left(v_{k}\right) \leq\left(n^{3}-2 n^{2}+3 n+6\right) / 4$.

We now discuss vertex exponents for the two-colored digraphs $L_{n}^{(2)}$ whose exponents is $2 n^{2}-6 n+2$.

Theorem 4.6. Let $L_{n}^{(2)}$ be a primitive two-colored digraph on $n \geq 5$ vertices whose underlying digraph is the digraph $L_{n}$ in Figure 1 . If $\exp \left(L_{n}^{(2)}\right)=2 n^{2}-6 n+2$, then for any vertex $v_{k}, k=1,2, \ldots, n$ we have $\left(n^{2}-4 n+5\right) \leq \gamma_{L_{n}^{(2)}}\left(v_{k}\right) \leq\left(n^{2}-2 n-1\right)$. 
Proof. Since $\exp \left(L_{n}^{(2)}\right)=2 n^{2}-6 n+2$, Corollary 2.4 implies that $L_{n}^{(2)}$ has a unique $(2,0)$-path that lies on both cycles. This implies the $(2,0)$-path of $L_{n}^{(2)}$ is of the form $a \rightarrow a-1 \rightarrow a-2$ for some $3 \leq a \leq n-2$. We show that $\gamma_{L_{n}^{(2)}}\left(v_{k}\right)=n^{2}-3 n+k+2-a$ for all $k=1,2, \ldots, n$. We first show that $\gamma_{D^{(2)}}\left(v_{k}\right) \geq n^{2}-3 n+k+2-a$ for all $k=1,2, \ldots, n$. We use path from $v_{k}$ to $v_{a-2}$ to determine the value of the quantity $u_{0}$ in equation (1) and we use path from $v_{k}$ to $v_{a}$ to determine the value of the quantity $v_{0}$ in equation (2). We split the proof into three cases depending on the position of the vertex $v_{k}$.

Case 1: $1 \leq k \leq a-2$

We note that there are two paths from $v_{k}$ to $v_{a-2}$. They are a $((n-1) / 2-\lfloor(a-2-$ $k) / 2\rfloor,(n-3) / 2-\lceil(a-2-k) / 2\rceil)$-path and a $((n+1) / 2-\lfloor(a-2-k) / 2\rfloor,(n-1) / 2-$ $\lceil(a-2-k) / 2\rceil)$-path. Using the first path and the definition of $u_{0}$ in equation (1) we have $u_{0}=1+\frac{n+1}{2}\left\lceil\frac{a-2-k}{2}\right\rceil-\frac{n-1}{2}\left\lfloor\frac{a-2-k}{2}\right\rfloor$. Using the second path and the definition of $u_{0}$ in equation (1) we have $u_{0}=\frac{n+1}{2}\left\lceil\frac{a-2-k}{2}\right\rceil-\frac{n-1}{2}\left\lfloor\frac{a-2-k}{2}\right\rfloor$. Hence we conclude that

$$
u_{0}=(n+1)\lceil(a-2-k) / 2\rceil / 2-(n-1)\lfloor(a-2-k) / 2\rfloor / 2 .
$$

There are two paths from $v_{k}$ to $v_{a}$. They are $\mathrm{a}((n-5) / 2-\lfloor(a-2-k) / 2\rfloor,(n-$ $3) / 2-\lceil(a-2-k)\rceil)$-path and a $((n-3) / 2-\lfloor(a-2-k) / 2\rfloor,(n-1) / 2-\lceil(a-2-k)\rceil)$ path. Using the first path and the definition of $v_{0}$ in equation (2) we have $v_{0}=$ $n-3-\frac{n-1}{2}\left\lceil\frac{a-2-k}{2}\right\rceil+\frac{n-3}{2}\left\lfloor\frac{a-2-k}{2}\right\rfloor$. Using the second path and the definition of $v_{0}$ in equation (2) we have $v_{0}=n-2-\frac{n-1}{2}\left\lceil\frac{a-2-k}{2}\right\rceil+\frac{n-3}{2}\left\lfloor\frac{a-2-k}{2}\right\rfloor$. Hence we conclude that

$$
v_{0}=n-3-(n-1)\lceil(a-2-k) / 2\rceil / 2+(n-3)\lfloor(a-2-k) / 2\rfloor .
$$

Now Theorem 3.2, equation (42) and equation (43) imply that

$$
\begin{aligned}
{\left[\begin{array}{l}
s \\
t
\end{array}\right] } & \geq M\left[\begin{array}{l}
u_{0} \\
v_{0}
\end{array}\right] \\
& =\left[\begin{array}{ll}
(n-1) / 2 & (n+1) / 2 \\
(n-3) / 2 & (n-1) / 2
\end{array}\right]\left[\begin{array}{r}
\frac{n+1}{2}\left\lceil\frac{a-2-k}{2}\right\rceil-\frac{n-1}{2}\left\lfloor\frac{a-2-k}{2}\right\rfloor \\
n-3-\frac{n-1}{2}\left\lceil\frac{a-2-k}{2}\right\rceil+\frac{n-3}{2}\left\lfloor\frac{a-2-k}{2}\right\rfloor
\end{array}\right] \\
& =\left[\begin{array}{l}
\left(n^{2}-2 n-3\right) / 2-\lfloor(a-k-2) / 2\rfloor \\
\left(n^{3}-4 n+3\right) / 2-\lceil(a-2-k) / 2\rceil
\end{array}\right]
\end{aligned}
$$

Hence we now have

$$
\begin{aligned}
\gamma_{D^{(2)}}\left(v_{k}\right) & \geq n^{2}-3 n-(\lfloor(a-2-k) / 2\rfloor+\lceil(a-2-k) / 2\rceil) \\
& =n^{2}-3 n+k+2-a
\end{aligned}
$$

for $k=1,2, \ldots, a-2$.

Case $2: k=a-1, a$

There is a unique path from $v_{k}$ to $v_{a-2}$ and it is a $(k-a+2,0)$-path. Using this path and the definition of $u_{0}$ in equation (1) we have that

$$
u_{0}=(n-1)(k-a+2) / 2 .
$$


There are two paths from $v_{k}$ to $v_{a}$. They are a $(k-a+2+(n-5) / 2,(n-3) / 2)$-path and a $(k+2-a+(n-3) / 2,(n-1) / 2)$-path. Using the first path and the definition of $v_{0}$ in equation (2) we have that $v_{0}=n-3-(n-3)(k+2-a) / 2$. Using the second path and the definition of $v_{0}$ in equation (2) we have that $v_{0}=n-2-(n-3)(k+2-a) / 2$. Hence we conclude that

$$
v_{0}=n-2-(n-3)(k+2-a) / 2 .
$$

Now Theorem 3.2, equation (46) and equation (47) imply that

$$
\begin{aligned}
\gamma_{D^{(2)}}\left(v_{k}\right) & \geq \ell\left(C_{1}\right) u_{0}+\ell\left(C_{2}\right) v_{0} \\
& =(n-2)(n-1)(k-a+2) / 2+n[(n-3)-(n-3)(k-a+2) / 2] \\
& =n^{2}-3 n+k+2-a
\end{aligned}
$$

for all $k=a-1, a$.

Case $3: a+1 \leq k \leq n$

There is a unique path from $v_{k}$ to $v_{a-2}$ which is $(\lfloor(k-a) / 2\rfloor+2,\lceil(k-a) / 2\rceil)$ path. Using this path and the definition of $u_{0}$ in equation (1) we have $u_{0}=$ $\left(\frac{n-1}{2}\right)\left(\left\lfloor\frac{k-a}{2}\right\rfloor+2\right)-\frac{n+1}{2}\left\lceil\frac{k-a}{2}\right\rceil$. There is a unique path from $v_{k}$ to $v_{a}$ which is a $(\lfloor(k-a) / 2\rfloor,\lceil(k-a) / 2\rceil)$-path. Using this path and the definition of $v_{0}$ in equation (2) we have that $v_{0}=\frac{n-1}{2}\left\lfloor\frac{k-a}{2}\right\rfloor-\frac{n-3}{2}\left\lceil\frac{k-a}{2}\right\rceil$. By Theorem 3.2 we have

$$
\begin{aligned}
\gamma_{L_{n}^{(2)}}\left(v_{k}\right) \geq & \ell\left(C_{1}\right) u_{0}+\ell\left(C_{2}\right) v_{0} \\
= & (n-2)\left(\frac{n-1}{2}\left(\left\lfloor\frac{k-a}{2}\right\rfloor+2\right)-\frac{n+1}{2}\left\lceil\frac{k-a}{2}\right\rceil\right) \\
& +n\left(\frac{n-1}{2}\left\lfloor\frac{k-a}{2}\right\rfloor-\frac{n+1}{2}\left\lceil\frac{k-a}{2}\right\rceil\right)
\end{aligned}
$$

Hence

$$
\begin{aligned}
& =n^{3}-3 n+2+\lfloor(k-a) / 2\rfloor+\lceil(k-a) / 2\rceil \\
& =n^{2}-3 n+k+2-a
\end{aligned}
$$

for $a+1 \leq k \leq n$.

From equation (45), equation (48) and equation (49) we conclude that

$$
\gamma_{L_{n}^{(2)}}\left(v_{k}\right) \geq n^{3}-3 n+k+2-a
$$

for all $k=1,2, \ldots, n$.

We now show that $\gamma_{L_{n}^{(2)}}\left(v_{k}\right) \leq n^{3}-3 n+k+2-a$ for $k=1,2, \ldots, n$. We first show that $\gamma_{L_{n}^{(2)}}\left(v_{1}\right) \leq n^{3}-3 n+3-a$ and then we use Proposition 3.4 to show that $\gamma_{L_{n}^{(2)}} \leq n^{2}-3 n+k+2-a$ for $k=2,3, \ldots, n$. By considering equation (44) it suffices to show that for each vertex $v_{i}, i=1,2, \ldots, n$ there is a walk $w_{1, i}$ from $v_{1}$ to $v_{i}$ with composition

$$
\left[\begin{array}{l}
r\left(w_{1, i}\right) \\
b\left(w_{1, i}\right)
\end{array}\right]=\left[\begin{array}{l}
\left(n^{2}-2 n-3\right) / 2-\lfloor(a-3) / 2\rfloor \\
\left(n^{3}-4 n+3\right) / 2-\lceil(a-3) / 2\rceil
\end{array}\right] .
$$


For each $i=1,2, \ldots, n$, let $p_{1, i}$ be a path from $v_{1}$ to $v_{i}$. We note that the solution to the system

$$
M\left[\begin{array}{l}
x_{1} \\
x_{2}
\end{array}\right]+\left[\begin{array}{l}
r\left(p_{1, i}\right) \\
b\left(p_{1, i}\right)
\end{array}\right]=\left[\begin{array}{l}
\left(n^{2}-2 n-3\right) / 2-\lfloor(a-3) / 2\rfloor \\
\left(n^{3}-4 n+3\right) / 2-\lceil(a-3) / 2\rceil
\end{array}\right]
$$

is the integer vector

$$
\begin{aligned}
{\left[\begin{array}{l}
x_{1} \\
x_{2}
\end{array}\right]=} & {\left[\begin{array}{c}
\lceil(a-3) / 2\rceil(n+1) / 2-\lfloor(a-3) / 2\rfloor(n-1) / 2 \\
n-3+\lfloor(a-3) / 2\rfloor(n-3) / 2-\lceil(a-3) / 2\rceil(n-1) / 2
\end{array}\right] } \\
+ & {\left[\begin{array}{l}
b\left(p_{1, i}\right)+\left(b\left(p_{1, i}\right)-r\left(p_{1, i}\right)\right)(n-1) / 2 \\
\left(r\left(p_{1, i}\right)-b\left(p_{1, i}\right)\right)(n-3) / 2-b\left(p_{1, i}\right)
\end{array}\right] . }
\end{aligned}
$$

We show that $x_{1} \geq 0$ and $x_{2} \geq 0$. We consider two cases when $a$ is even and $a$ is odd. implies

If $a$ is even, then $\lceil(a-3) / 2\rceil=(a-2) / 2$ and $\lfloor(a-3) / 2\rfloor=(a-4) / 2$. This

$$
\left[\begin{array}{l}
x_{1} \\
x_{2}
\end{array}\right]=\left[\begin{array}{c}
(n-1) / 2+(a-2) / 2+b\left(p_{1, i}\right)-\left[r\left(p_{1, i}\right)-b\left(p_{1, i}\right)\right](n-1) / 2 \\
(n-a-1) / 2+\left[r\left(p_{1, i}\right)-b\left(p_{1, i}\right)\right](n-3) / 2-b\left(p_{1, i}\right)
\end{array}\right] .
$$

Since $a$ is even, we have that $0 \leq r\left(p_{1, i}\right)-b\left(p_{1, i}\right) \leq 2$. If $r\left(p_{1, i}\right)-b\left(p_{1, i}\right)=0$, then $b\left(p_{1, i}\right) \leq(n-1-a) / 2$. This implies $x_{1}>0$ and $x_{2} \geq 0$. If $r\left(p_{1, i}\right)-b\left(p_{1, i}\right)=1$, there is a path $p_{1, i}$ with $b\left(p_{1, i}\right) \leq(n-3) / 2$. This implies $x_{1}>0$ and $x_{2}>0$. If $r\left(p_{1, i}\right)-b\left(p_{1, i}\right)=2$, then $b\left(p_{1, i}\right) \geq(n+1-a) / 2$. This implies $x_{1} \geq 0$ and $x_{2}>0$. Therefore for each vertex $v_{i}, i=1,2, \ldots, n$, there is a path $p_{1, i}$ from $v_{1}$ to $v_{i}$ such that the system (52) has nonnegative integer solution $x_{1} \geq 0$ and $x_{2} \geq 0$.

If $a$ is odd, then $\lceil(a-3) / 2\rceil=\lfloor(a-3) / 2\rfloor=(a-3) / 2$. This implies

$$
\left[\begin{array}{l}
x_{1} \\
x_{2}
\end{array}\right]=\left[\begin{array}{c}
(a-3) / 2+b\left(p_{1, i}\right)-\left[r\left(p_{1, i}\right)-b\left(p_{1, i}\right)\right](n-1) / 2 \\
n-3-(a-3) / 2+\left[r\left(p_{1, i}\right)-b\left(p_{1, i}\right)\right](n-3) / 2-b\left(p_{1, i}\right)
\end{array}\right] .
$$

Since $a$ is odd, for each vertex $v_{i}, i=1,2, \ldots, n$ there is a path $p_{1, i}$ with $-1 \leq$ $r\left(p_{1, i}\right)-b\left(p_{1, i}\right) \leq 1$. If $r\left(p_{1, i}\right)-b\left(p_{1, i}\right)=-1$, there is a path $p_{1, i}$ with $b\left(p_{1, i}\right) \leq$ $(n-a) / 2$. This implies $x_{1}>0$ and $x_{2} \geq 0$. If $r\left(p_{1, i}\right)-b\left(p_{1, i}\right)=0$, there is a path $p_{1, i}$ with $b\left(p_{1, i}\right) \leq(n-3) / 2$. This implies $x_{1}>0$ and $x_{2}>0$. Finally if $r\left(p_{1, i}\right)-b\left(p_{1, i}\right)=1$, there is a path $p_{1, i}$ with $b\left(p_{1, i}\right) \geq(n-a+2) / 2$. This implies $x_{1} \geq 0$ and $x_{2}>0$. Therefore for each vertex $v_{i}, i=1,2, \ldots, n$, there is a path $p_{1, i}$ from $v_{1}$ to $v_{i}$ such that the system (52) has nonnegative integer solution $x_{1} \geq 0$ and $x_{2} \geq 0$.

Since the system (52) has a nonnegative integer solution and the vertex $v_{1}$ belongs to both cycles, Proposition 3.3 guarantees that $\gamma_{L_{n}^{(2)}}\left(v_{1}\right) \leq n^{2}-3 n+3-a$. Combining this with equation (45) we conclude that $\gamma_{L_{n}^{(2)}}^{\left(v_{1}\right)}=n^{2}-3 n+3-a$. Since for $k=2,3, \ldots, n$ we have $d\left(v_{k}, d_{1}\right)=k-1$, Proposition 3.4 implies that

$$
\gamma_{L_{n}^{(2)}}\left(v_{k}\right) \leq n^{2}-3 n+k+2-a
$$

for $k=1,2, \ldots, n$.

Finally combining equation (50) and equation (54) we conclude that $\gamma_{L_{n}^{(2)}}\left(v_{k}\right)=$ $n^{2}-3 n+k+2-a$ for $k=1,2, \ldots, n$. We note that $3 \leq a \leq n-2$. This implies 
$n^{2}-4 n+4+k \leq \gamma_{L_{n}^{(2)}}\left(v_{k}\right) \leq n^{2}-3 n+k-1$. Therefore for any $k=1,2, \ldots, n$ we have $n^{2}-4 n+5 \leq \gamma_{L_{n}^{(2)}}\left(v_{k}\right) \leq n^{2}-2 n-1$.

\section{References}

[1] Fornasini, E. and Valcher, M. E., "Primitivity positive matrix pairs: algebraic characterization graph theoritic description and 2D systems interpretations", SIAM J. Matrix Anal. Appl. 19 (1998), 71-88

[2] Gao, Y. and Shao, Y., "Exponents of two-colored digraphs with cycles", Linear Algebra Appl. 407 (2005), 263-276

[3] Gao, Y. and Shao, Y., "Exponents of a class two-colored digraphs", Linear and Multilinear Algebra 53 (3) (2005), 175-188

[4] Gao, Y. and Shao, Y., "Generalized exponents of primitive two-colored digraphs", Linear Algebra Appl. 430 (2009), 1550-1565

[5] Lee, S. G. and Yang, J. M., "Bound for 2-exponents of primitive extremal ministrong digraphs", Commun. Korean. Math. Soc. 20 no. 1 (2005), 51-62

6] Huang, F. and Liu, B., "Exponents of a class of two-colored digraphs with two cycles", Linear Algebra and Appl. 429 (2008), 658-672

[7] Shader, B. L. and Suwilo, S., "Exponents of nonnegative matrix pairs", Linear Algebra Appl. 263 (2003), 275-293

[8] Suwilo, S., On 2-exponents of 2-digraphs, Ph.D. Dissertation, University of Wyoming, 2001.

[9] Suwilo, S., "Vertex Exponents of two-colored primitive extremal ministrong digraphs", Global Journal of Technology and Optimization, Vol 2 No 2 (2011), 166-174 